\title{
LA ACCIÓN SONORA COMO REIVINDICACIÓN SOCIAL: UNA INTERVENCIÓN ARTÍSTICA EN EL BARRIO ESPÍRITU SANTO DE ESPINARDO (MURCIA)
}

\author{
Pedro Ortuño Mengual \\ Gloria Lapeña Gallego ${ }^{1}$ \\ Departamento de Bellas Artes. Grupo de Investigación Arte y Políticas de Identidad. \\ Universidad de Murcia. Campus Universitario Espinardo.
}

\section{Resumen}

El objetivo de este trabajo es analizar una práctica colaborativa de intervención artística, Ilevada a cabo por alumnos de la asignatura optativa de Grado de Bellas Artes de la Universidad de Murcia, "Intervenciones escultóricas en el espacio urbano y natural". En un barrio marginal de Murcia, los estudiantes y las familias, tras una serie de encuentros, acuerdan por consenso remodelar una de las calles. Inspirados en el manifiesto futurista de Russolo (1913), ponen especial atención en la escucha de sonidos, ruidos y palabras habladas de las calles. Las propuestas resultantes convergen en la seña de identidad ligada al cante, toque y baile flamenco que materializan en rehabilitación de fachadas, mejora de infraestructuras y pintura mural como lugar en el que se hibrida la idiosincrasia étnica y la práctica artística.

Palabras clave: ARTE PÚBLICO; ACCIÓN SONORA; ARTE COLABORATIVO; INTERVENCIÓN ARTÍSTICA; REIVINDICACIÓN SOCIAL

\section{THE SOUND ACTION AS SOCIAL DEMANDS: AN ARTISTIC INTERVENTION IN THE BARRIO ESPÍRITU SANTO DE ESPINARDO (MURCIA)}

Abstract

The aim of this paper is to analyze a collaborative practice of artistic intervention, carried out by students of the elective course in Fine Arts degree from the University of Murcia, "Sculptural interventions in the urban and natural space". In a slum in Murcia, students and families, after a series of meetings, agreed by consensus to remodel one of the streets. We were inspired by the Futurist Manifesto of Russolo (1913) and attend in listening sounds, noises and words spoken in the streets. The resulting proposals converge on the hallmark linked to singing, playing and dancing flamenco embodying rehabilitation of facades, improvement of infrastructure and mural painting as a place where ethnic and idiosyncratic artistic practice hybridizes.

Keywords: PUBLIC ART; SOUND ACTION; COLLABORATIVE ART; ARTISTIC INTERVENTION; SOCIAL CLAIM

\footnotetext{
Ortuño Mengual, Pedro \& Gloria Lapeña Gallego. 2015. "La acción sonora como reivindicación social: Una intervención artística en el Barrio Espíritu Santo de Espinardo". AusArt 3 (2): 98-105. D0I: 10.1387 /ausart.15940
}

\section{AUSART}




\section{INTRODUCCIÓN}

El espacio urbano, continuamente apropiado por la sociedad que lo habita, puede ser abordado desde un ámbito multidisciplinar más amplio que la ciudad, objeto de estudio de arquitectos y urbanistas (Delgado 2007). En el área de las artes visuales los estudios sobre el espacio urbano se orientan hacia intervenciones en las que la obra se constituye como objeto y como acto perceptivo. La colaboración ciudadana resulta una pieza clave en la elaboración de un proyecto que se concibe como específico para el lugar y con arreglo a la identidad de sus habitantes.

Los actuales estudios de Grado de Bellas Artes de la Universidad de Murcia incluyen una serie de asignaturas optativas que completan las obligatorias y/o presentan contenidos por los que el alumno puede tener especial inclinación con vistas a su futuro profesional. "Intervenciones escultóricas en el espacio urbano y natural" es una de las asignaturas optativas, se imparte en el primer cuatrimestre de $4^{\circ}$ curso y tiene una duración de 6 créditos.

Durante el curso académico 2014-15, para el módulo de intervención escultórica en el espacio urbano, planteamos una práctica colaborativa en el barrio Espíritu Santo de Espinardo perteneciente al municipio de Murcia. Inspirada en el manifiesto futurista de Luigi Russolo de 1913, los alumnos toman contacto con los vecinos y aprenden a escuchar sonidos, ruidos y conversaciones, a modo de partitura musical que contiene una multiplicidad de melodías y sonidos con un dominio de todo lo relacionado con el flamenco. Considerando el arte sonoro como obras constituidas preferentemente por sonidos naturales o tecnológicos desarrollados dentro de diferentes lenguajes propios del arte contemporáneo, entre ellos la escultura, no podemos afirmar que las intervenciones resultantes sean arte sonoro. Sin embargo, puesto que hemos centrado la atención en las manifestaciones del fenómeno sonoro como acción para "escuchar" y poder introducir a los destinatarios del proyecto también como partícipes y colaboradores que reivindican una vida de integración acorde con la sociedad contemporánea, estamos actuando como receptores de sonidos para materializarlo en una intervención en el espacio urbano.

El objetivo de este artículo es analizar estas intervenciones y las propuestas resultantes de trasfondo reivindicativo a partir de una audición activa como base metodológica de investigación previa para conocer las identidades de los habitantes del barrio. La hipótesis de trabajo es la utilización del arte sonoro como investigación y acción a lo largo del proyecto artístico para la elabora- 
ción de una intervención artística en un lugar concreto del espacio urbano. Para estructurar el texto estableceremos, en primer lugar, las bases teóricas en las que fundamentamos la práctica. A continuación explicaremos cómo se llevó a cabo la misma y las herramientas teórico prácticas de intervención que utilizan los alumnos en el espacio urbano a través de una metodología de investigación social cualitativa. Por último extraeremos las conclusiones en base al cumplimiento de nuestra hipótesis de trabajo.

\section{BASES TEÓRICAS}

El catálogo de la exposición Sound/Art comisariada por William Hellerman (The Sculpture Center, New York, 1982) incluye un ensayo que resume las características que engloba el término arte sonoro. Hellermann apunta que la escucha es otra forma de ver y el historiador Don Goddart considera que la conjunción del sonido y de la imagen insiste en la implicación del espectador, forzando su participación en el espacio real y concreto, en lugar de responder a un espacio imaginado y pensado (Hellerman \& Goddard 1983). No es la primera vez que se relaciona la percepción visual con la auditiva. Cabe destacar varias exposiciones bajo el título "Para los ojos y los oídos". For Eyes \& Ears, en la Cordier \& Ekstrom Gallery de New York en 1964 con objetos sonoros dadaístas de Marcel Duchamp y Man Ray, audio-instalaciones de Klüver y Rauschenberg, obras cinéticas sonoras de Jean Tinguely y Takis; Für Augen und Ohren, en la Akademie der Künste de Berlin; Écouter par les yeux en el Musée d'Art Moderne de la Ville de Paris. Estas últimas, ambas de 1980, incluyen objetos sonoros, instalaciones, performances y los conceptos entornos acústicos o ambientes sonoros (Molina Alarcón 2008).

Estas obras tienen en común, además de la doble percepción auditiva- visual, que son piezas creadas para un espacio expositivo. Sin embargo, dentro del arte sonoro, los paisajes sonoros parten de metodologías de estudios y análisis de aspectos sociales, culturales y artísticos de un lugar específico (Palmese, Carles \& Alcázar 2010). Así, Ariza-Pomareta (2015), con motivo de la llegada del tren de alta velocidad a Cuenca en 2010, construye un paisaje sonoro con los rasgos de la comunicación ferroviaria de esta ciudad desde 1883 hasta la primera década del siglo XXI. Según este artista-investigador, la grabación de los sonidos está sujeta a variables subjetivas que van a depender de factores como el momento, lugar, calidad y formato de la captura del sonido. Por tanto, 
la construcción de un paisaje sonoro origina un relato subjetivo no real consecuencia de la suma de interpretaciones (Iges 2001).

El origen del arte sonoro se remota a las vanguardias del principios del siglo XX con el manifiesto Futurista L'Arte dei rumori o "El arte de los ruidos" de Luigi Russolo publicado en Milán el 11 de marzo de 1913. Plantea que la llegada de la vida moderna viene acompañada con el ruido como símbolo de progreso humano. El ruido se enfrenta así al sonido del mundo antiguo que es el silencio, atribuido a los dioses y reservado a los sacerdotes. La música es consecuencia de esta idea de sonido, algo independiente de la vida, por encima de la realidad y en un plano sagrado. El ruido es por tanto una necesidad del oído tras la evolución de la música desde los sistemas griegos y cantos gregorianos hasta los acordes disonantes de la música contemporánea (Russolo 1998).

A lo largo del siglo $X X$, la figura del artista va adquiriendo un rol importante como agente del cambio social, a partir de las discusiones y acciones vanguardistas en torno al contenido político del arte. Aparecen entonces nuevos paradigmas estéticos y culturales con una clara función social: el espacio público se convierte en un lugar de intervención que está en relación directa con el arte y su contexto local y el espectador cobra un papel fundamental en el proceso de creación. Considerando como antecedente la teoría y práctica del Bertolt Brecht, que se articula entre el arte, la obra teatral y la praxis social y resultaba de la producción de un arte de masas que contribuía a desenmascarar la ideología de la clase dominante e intentaba concienciar sobre la desigualdad social. Para Ranciére, en su libro el espectador emancipado, la visión del espectáculo no tiene como final simplemente la comprensión intelectual o la toma de conciencia del mundo; a partir de ahí, el espectador debía dedicarse a actuar, pasando así a otra esfera: de la visión sensible del mundo a otra que define otras visiones de tolerancia e intolerancia, capacidades e incapacidades. Se supone que el arte es político porque muestra los estigmas de la dominación, o bien porque pone en ridículo los iconos reinantes o incluso porque sale de los lugares que les son propios para transformarlos en práctica social (Ranciére 2010, 54). Y partiendo de esa perspectiva el espectador acabaría reconociendo la posibilidad de realizar una interpretación activa y no solamente emancipatoria en la que el valor político del arte no se encuentra en el contenido, sino en las formas del proceso que lo hace visible. La emancipación en el arte pasaría por el reconocimiento de que la producción de la obra no pertenece a quien la crea. De esta forma, entendemos el arte colaborativo como un arte que intenta que se revise el papel del artista, del público y de la obra, cuestionando así la concepción tradicional del arte. El artista se coloca 
en situación de igualdad con el espectador, y el espectador deja de ser el receptor pasivo y pasa a integrarse activamente con el desarrollo de la obra.

\section{PLANIFICACIÓN DEL PROYECTO}

Aprender haciendo es una modalidad pedagógica donde los estudiantes, organizados en pequeños grupos de aprendizaje, aprenden mediante la resolución de problemas y la realización de proyectos. Se organizan "en torno de un problema concreto cuya responsabilidad de ejecución está a cargo de un equipo integrado por profesores y alumnos que participan activa y responsablemente en todas las fases o etapas de realización" (Ander-Egg 1999) y los conocimientos se adquieren en una práctica concreta que implica la inserción en la realidad. Tratamos de construir puentes entre el conocimiento teórico y el práctico. Para construir estos puentes de aprendizaje además introducimos nuevos elementos relacionados con el aprendizaje colaborativo. De esta forma se intenta fomentar la colaboración y las relaciones de igualdad creando un entorno de aprendizaje en el cual cada alumno no solo es responsable de su aprendizaje sino que también contribuye al de los demás. Los estudiantes se ayudan mutuamente, ya que tanto el que explica como el que aprende alcanza una mejor comprensión de la materia.

El barrio de Espíritu Santo en Espinardo, es uno de los más marginales de la Región de Murcia, donde sus vecinos son mayoritariamente de etnia gitana. Actualmente es un lugar que se caracteriza por su situación de riesgo social, su diversidad cultural y su carácter controvertido, al ser un núcleo donde se concentra la venta de drogas en la ciudad. El proyecto se plantea como una estrategia de intervención artística en un espacio público muy deteriorado por el mal uso que se hace de él. Con posterioridad a una serie de encuentros entre los estudiantes y los habitantes del barrio.

El proceso de construcción de la obra tuvo lugar a partir de diversas reuniones con los vecinos del barrio. A lo largo de las mismas tuvimos la oportunidad de invadir el terreno de lo privado de alguna de las casas, en las que se nos invitaba a pasar y escuchar el bullicio de la vida de esta gente, con profundos ecos de sentimientos en su expresión a todos los niveles. Los alumnos realizaron muchas grabaciones de este alboroto sonoro de palabras, gestos y expresiones. 


\section{RESULTADOS}
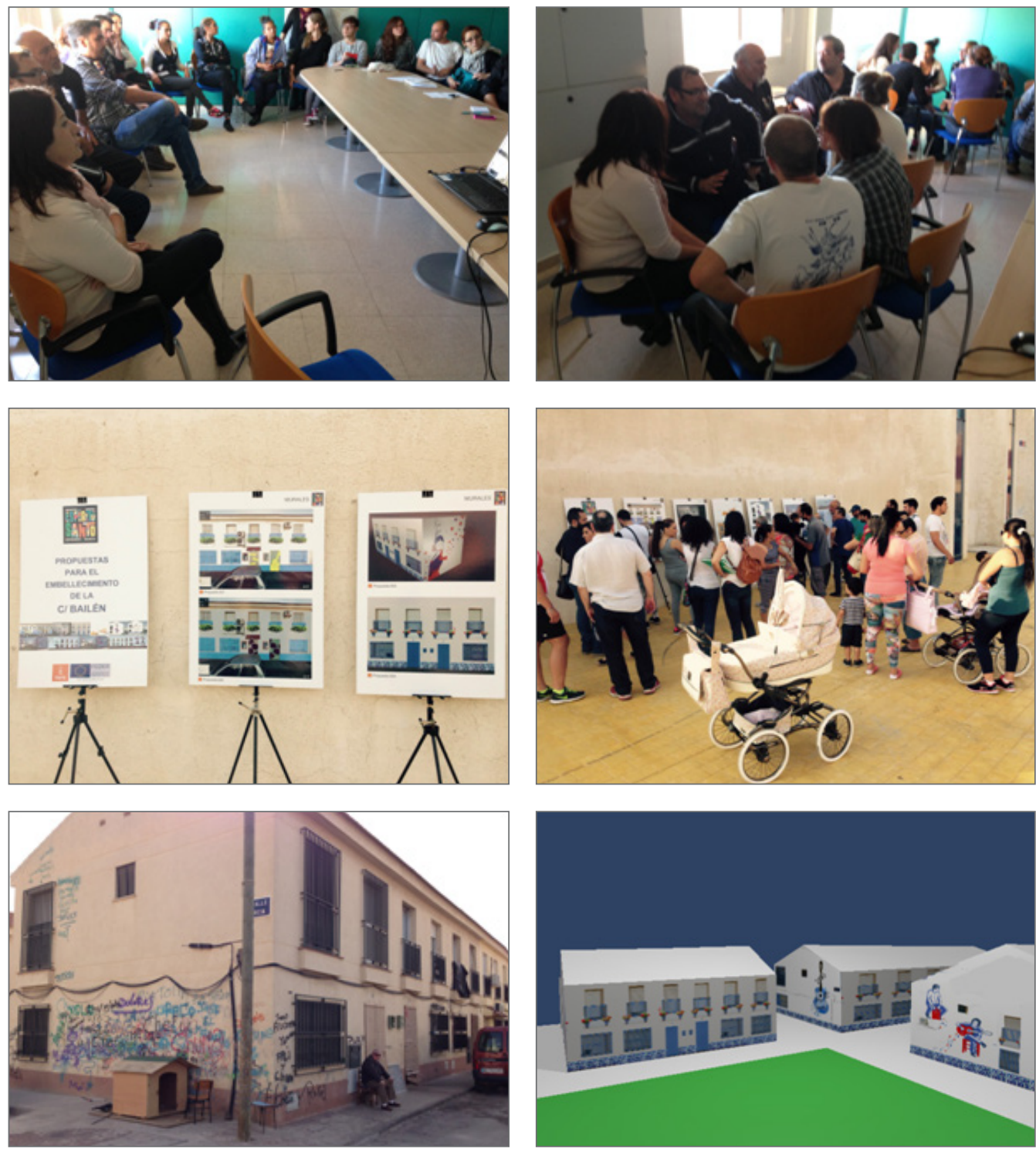

Figuras 1-6: Reuniones con los vecinos del barrio Espíritu Santo (Espinardo, Murcia), trípodes con paneles de los siete proyectos planteados y ubicación y diseño de los murales. 
Con toda esta idiosincrasia de sonidos, este colectivo reivindica una vida normalizada dentro de la ciudad, un barrio menos deteriorado, una mayor dotación de servicios dentro del mismo, unos parques infantiles con dotación de columpios, toboganes... donde puedan jugar sus hijos. En definitiva que el barrio de Espíritu Santo se pueda equiparar en igualdad de condiciones a cualquier otro barrio de la ciudad de Murcia.

Con esta modalidad pedagógica de aprender haciendo, las reuniones se sucedieron en un periodo de seis meses, de tal forma que pudimos conocer a gran parte de los vecinos del barrio los cuales participaron de forma directa en el trazado de las ideas que sustentaban cada uno de los proyectos que se gestaron. Finalmente había que decidirse por uno y para facilitar esta toma de decisiones se instalaron siete trípodes en la calle, para mostrar en unos paneles la expresión gráfica de los proyectos con el fin de que cada uno de los vecinos pudiera elegir y opinar acerca del que más le gustaba. Por último se decidió realizar un híbrido de dos de los proyectos expuestos, ambos destinados a la mejora estética del barrio. Los elementos sonoros de los proyectos, que tienen que ver con los sonidos particulares de este barrio gitano y el cante flamenco de sus gentes, se plasmaron a nivel plástico mediante la realización de cuatro pinturas murales y grabado o serigrafía impresa. Estas pinturas murales llevan implícito la restauración de todas las fachadas de las viviendas de la calle así como el compromiso del ayuntamiento de mejora de sus infraestructuras.

El proyecto de la calle Bailén se convierte así en una propuesta artística que parte del fenómeno sonoro como acción para escuchar y recoge los sonidos del barrio para transformarlos en un icono de identidad de sus habitantes, un lugar en continua transformación, un espacio peculiar donde los vecinos habitualmente pasan mucho tiempo en la calle por la noche con sus guitarras, sus tertulias, sus bailes y su cante.

\section{A MODO DE CONCLUSIÓN}

Se puede decir a modo de conclusión, que el paisaje sonoro del colectivo gitano anclado en el barrio del Espíritu Santo, podría ser entendido como el espacio de vida que rodea a cada uno de sus vecinos, en el que se incluye lo que afecta a cada uno individualmente y lo que afecta como miembro de este colectivo de pertenencia. A esto habría que añadir el reconocimiento en cada 
una de las propuestas de los alumnos de alguna de las señas de identidad cultural del entorno, su interés y disposición favorable para entablar relaciones respetuosas y afectivas con este colectivo gitano y su acercamiento a sus costumbres y señas de identidad asociado a la realización de un trabajo plástico que recoge los sonidos del barrio.

\section{Referencias}

Acconci, Vito et al. 1983. Sound/Art. Catálogo de la exposición (New York, Sculpture Center, May 1-30 and BACA/DCC Gallery, June 1-30). Essay by Don Goddard; curated by William Hellermann. New York: SoundArt Foundation

Ander Egg, Ezequiel. 1999. El taller: Una alternativa de renovación pedagógica. Buenos Aires: Magisterio del Río de la Plata

Ariza Pomareta, Javier. 2015. "El paisaje sonoro como cápsula del tiempo: Un proyecto creativo basado en los sonidos de la primera estación de trenes de Cuenca". Arte, Individuo y Sociedad 27: 99-113

Delgado Ruiz, Manuel. 2007. Sociedades movedizas. Barcelona: Anagrama Iges, José. 2001. "Un approcio alla storia del paesaggio sonoro". Musica/Realtà 65: 55-66

Molina Alarcón, Miguel. 2008. "El arte sonoro". Itamar, Revista de Investigación Musical: Territorios para el Arte 1: 213-34

Palmese, Cristina, José Luis Carles Arribas \& Antonio Jesús Alcázar Aranda. 2010. Paisajes sonoros de Cuenca. Cuenca: UCLM

Rancière, Jacques. 2010. El espectador emancipado. Buenos Aires: Ellago

Russolo, Luigi. 1998. El arte de los ruidos. Cuenca: Centro de Creación Experimental UCLM

\section{Notas}

${ }^{1}$ Doctoranda con una ayuda predoctoral financiada (19099/FPI/13) con cargo al Programa de Formación del Personal Investigador de la Fundación Séneca, Agencia de Ciencia y Tecnología de la Región de Murcia en el marco del III PCTRM 2011-14, bajo la dirección y tutela del Dr. Ortuño Mengual. 